\title{
O PROBLEMA DE FLAVIUS JOSEPHUS
}

\author{
THE PROBLEM OF FLAVIUS JOSEPHUS
}

\section{Felippe Calsavara Gonçalves; Fernando Pereira de Souza.}

Universidade Federal de Mato Grosso do Sul, Departamento de Ciências Exatas

E-mail: pepecalsvara@gmail.com, fermatmel@gmail.com

RESUMO - Neste trabalho apresentamos uma solução para o problema proposto pelo matemático Flavius Josephus, no primeiro século depois de Cristo. De acordo com lenda, um grupo de rebeldes, dentre eles Josephus, foram encurralados em uma caverna pelo exército inimigo. Preferindo o suicídio à captura, os rebeldes decidiram formar um círculo e o primeiro deveria matar o soldado diretamente ao seu lado, assim até sobrar apenas um. A única solução seria fazer as contas e descobrir qual a posição do soldado que iria sobreviver. A solução pode ser obtida resolvendo o problema passo a passo, mas se o número de soldados for muito grande então a contagem fica inviável. Para encontrar tal solução com um número arbitrário de soldados, iniciamos nosso estudo com alguns conceitos de potenciação, recorrências e indução finita. Em seguida, introduzimos alguns conceitos de números binários e suas operações. Este artigo é parte das pesquisa elaborada no grupo PET Matemática da Universidade Federal de Mato Grosso do Sul no Campus de Três Lagoas.

Palavras-chave: Josephus; indução finita; números binários; potências.

ABSTRACT- In this work we present a solution to the problem proposed by the mathematician Flavius Josephus, in the first century after Christ. According to legend, a group of rebels, among them Josephus, were trapped in a cave by the enemy army. Preferring suicide to capture, the rebels decided to form a circle and the first one should kill the soldier directly to his side, thus until only one remains. The only solution would be to do the math and figure out the position of the soldier who would survive. The solution can be obtained solving the problem step by step, but if the number of soldiers is very large then the count becomes impracticable. To find such a solution with an arbitrary number of soldiers, we began our study with some concepts of potentiation, recurrence, and finite induction. Next, we introduce some concepts of

Recebido em: 09/08/2018 Revisado em: 03/09/2018 Aprovado em: 13/09/2018 binary numbers and their operations. This article is part of the research elaborated in the PET Mathematics group of the Federal University of Mato Grosso do Sul at Três Lagoas Campus.

Keywords: Josephus; finite induction; binary numbers; powers. 


\section{INTRODUÇÃO}

Flavius Josephus nasceu em Jerusalém, era de família sacerdotal, foi criado na melhor tradição da Judéia e recebeu boa educação geral. Segundo sua autobiografia, de onde procedem todas as informações a seu respeito, o pai ministrouIhe minuciosa iniciação nos textos tradicionais e legais da Torá. O historiador judeu do primeiro século depois de Cristo, lutou durante o cerco de Yodfat. Ele e outros 40 homens foram rendidos pelo exército romano, e optaram pelo suicídio para não se entregarem. Como tirar a própria vida não é fácil, ficou decidido então que o primeiro soldado vivo deveria matar o outro ao seu lado e assim por diante sempre na ordem. 0 último a matar ficaria vivo, Josephus não tinha muito apreço pela ideia de morrer. Então ele calculou rapidamente a posição adequada onde deveria ficar no círculo para sair ileso nessa situação. No decorrer deste trabalho, apresentamos uma solução para o Problema de Josephus, utilizando demonstrações por indução finita e apresentamos uma solução baseando-se no conceito de números binários.

\section{METODOLOGIA}

Pesquisas bibliográficas sobre 0 problema de Josephus e um estudo teórico sobre indução finita e números binários foram realizados. Foram feitas também apresentações com o orientador para uma melhor compreensão do assunto abordado.

\section{RESULTADOS}

O problema de Josephus aqui demonstrado baseia-se no princípio de indução finita. O método da indução finita é um procedimento matemático para provar propriedades que são verdadeiras para uma sequência de objetos. É um método bastante utilizado em teoria dos números, geometria, análise combinatória, etc....

\subsection{Princípio da Indução Finita:}

Seja $P(n)$ uma proposição que descreve uma propriedade sobre um número natural $n$. Suponhamos que as afirmações abaixo estejam satisfeitas:

i) $P(1)$ é verdadeiro;

ii) Se $P(2), \cdots, P(k-1)$ são verdadeiros, então $P(k)$ também é verdadeiro.

Então $P(n)$ é verdadeiro para todo número natural $n$.

Exemplo 1: Todo número natural maior do que 1 ou é primo ou se escreve de modo único como um produto de números primos.

Vamos denotar por $P(n)$ a afirmação: " $n$ se escreve de modo único como produto de primos exceto pela ordem dos fatores". É claro que $P(2)$ é verdadeiro, pois 2 é primo. Suponhamos que $P(k)$ seja verdadeira, para todo $2 \leq k<n$. Se $n$ for primo, não há o que fazer, $P(n)$ é verdadeira. Suponha que $n$ não seja primo, logo existe um número $p_{1}$ primo tal que $p_{1} \mid \mathrm{n}$, ou seja,

$$
n=p_{1} q \text {, }
$$

onde $q \in \mathbb{Z}$ e $2 \leq q<n$. Pela hipótese de indução finita, existem $p_{2} \ldots p_{r}$ primos maiores que zero tal que, $q=p_{2} \ldots p_{r}$. Assim, $n=p_{1} q=p_{1} . p_{2} \ldots p_{r}$, provando que $n$ pode ser escrito como produtos de primos.

\subsection{Problema de Josephus}

A sobrevivência de Josephus dentre os companheiros originou um curioso problema muito difundido, principalmente entre os matemáticos e que, mais geralmente, pode ser enunciado da seguinte maneira: Decide-se eliminar pessoas de um grupo da seguinte forma:

i) as pessoas são colocadas em um círculo com lugares marcados em ordem crescente no sentido horário, $(1,2,3, \ldots, n)$,

ii) este círculo é percorrido no sentido horário tantas vezes quanto necessário, começando com a pessoa no lugar 1 , e toda segunda 
pessoa viva nesta visitação é eliminada até que só uma sobreviva.

Qual a posição que a sobrevivente ocupa? (SANTOS; MELLO; MURARUI 2007, p. 234).

Consideramos neste trabalho que temos $n$ soldados e que $J(n)$ seja a posição do soldado que permanecerá vivo.

Exemplos:

$$
N=3 \text { temos } J(3)=3
$$

Figura 1. Caso $n=3$

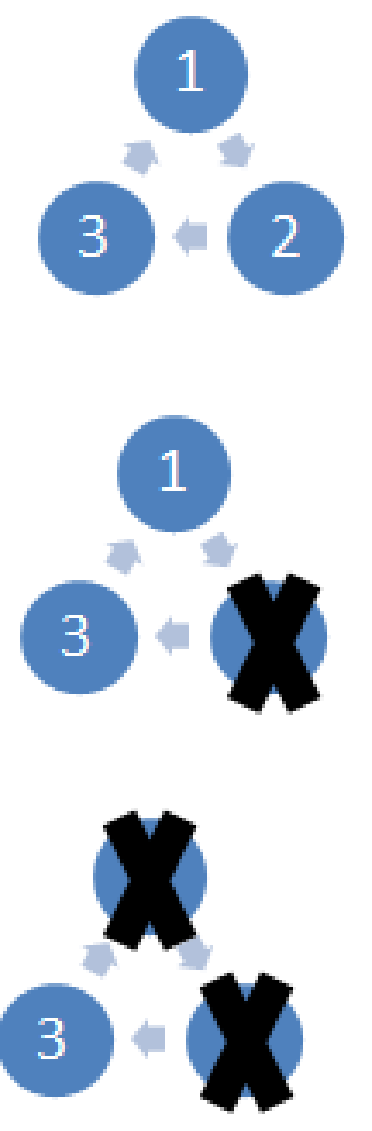

Fonte: $\mathrm{O}$ autor

$$
N=4 \operatorname{temos} J(4)=1
$$

Figura 2. Caso $n=4$

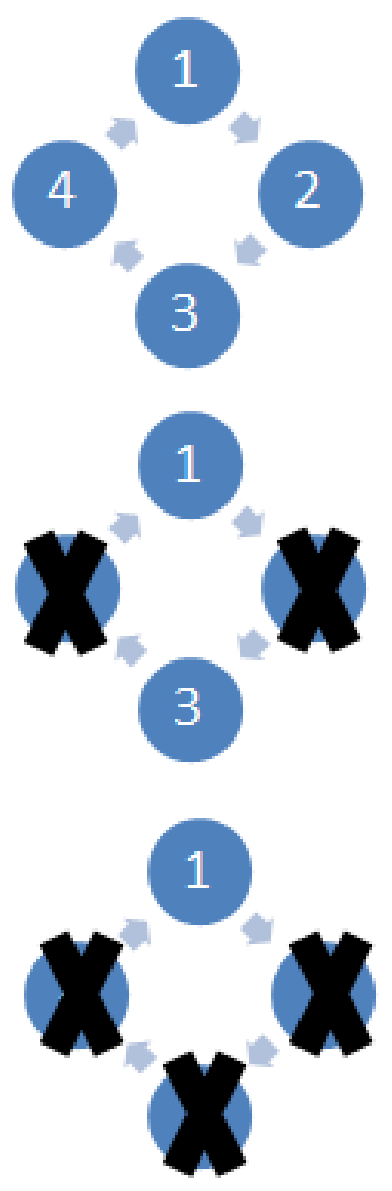

Fonte: $\mathrm{O}$ autor

Para alguns números pequenos temos:

Tabela 1. Valores pequenos para $\boldsymbol{n}$

\begin{tabular}{|c|c|}
\hline $\boldsymbol{n}$ & $\boldsymbol{J}(\boldsymbol{n})$ \\
\hline 1 & 1 \\
\hline 2 & 1 \\
\hline 3 & 3 \\
\hline 4 & 1 \\
\hline 5 & 3 \\
\hline 6 & 5 \\
\hline 7 & 7 \\
\hline 8 & 1 \\
\hline 9 & 3 \\
\hline 10 & 5 \\
\hline 11 & 7 \\
\hline 12 & 9 \\
\hline
\end{tabular}

Fonte: $\mathrm{O}$ autor 
A tabela (1) nos induz a conjecturar que:

$$
\begin{aligned}
J(1) & =1 \\
J(2 n) & =2 J(n)-1, \text { para } n \geq 1, \\
J(2 n+1) & =2 J(n)+1, \text { para } n \geq 1 .
\end{aligned}
$$

As equações (1) - (3) são válidas. De fato, considere $2 n$ soldados em um círculo,

Figura 3. Caso $\mathbf{2} \boldsymbol{n}$ soldados

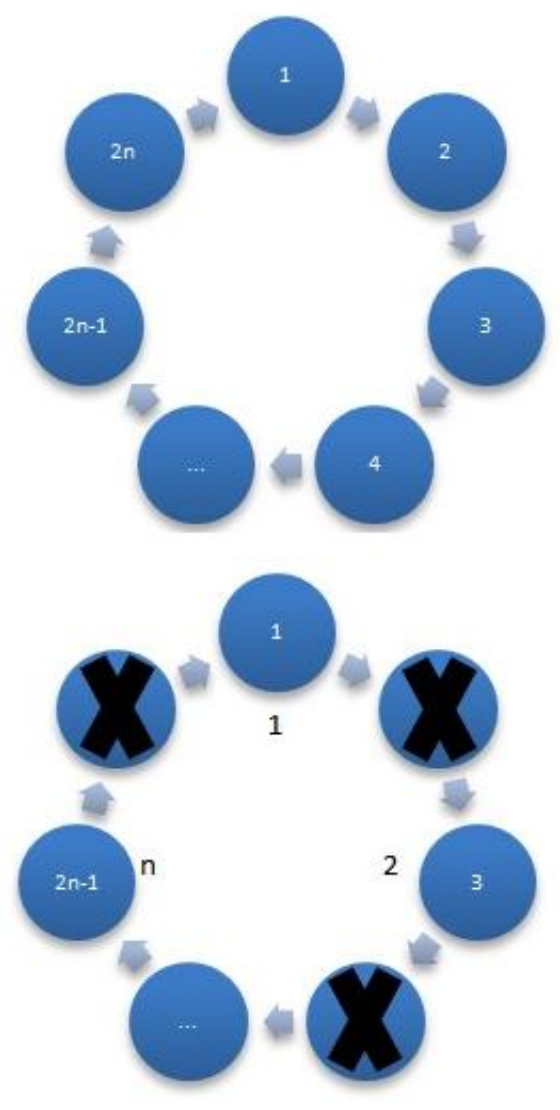

Fonte: $\mathrm{O}$ autor

Após a primeira rodada de eliminação, os soldados que estavam nas posições pares são eliminados, o primeiro soldado a começar a segunda rodada será o soldado de posição 1 e a quantidade de soldados restantes é $n$.

Observe que, após a primeira rodada temos novas posições para os soldados como mostra a tabela a seguir:
Tabela 2. Posições após a primeira rodada

\begin{tabular}{l|cccccc} 
Posição: $k$ & 1 & 3 & 5 & 7 & $\ldots$ & $2 n-1$ \\
\hline Nova posição: $k^{\prime}$ & 1 & 2 & 3 & 4 & $\ldots$ & $n$ \\
Fonte: O autor & & & & & &
\end{tabular}

ou ainda, $k=2 k^{\prime}-1$. A correspondência "posição" e "nova posição" nos dá

$$
J(2 n)=2 J(n)-1 \text {. }
$$

Suponhamos que temos $2 n+1$ soldados em um círculo.

Figura 4. Caso $2 n+1$ soldados

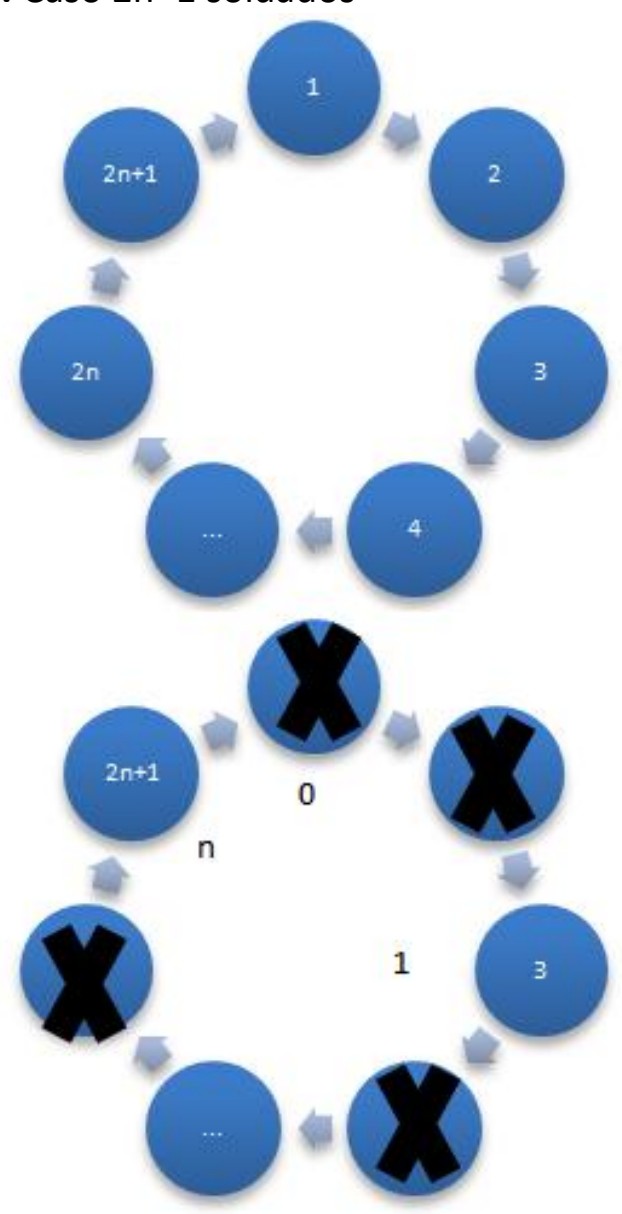

Fonte: $\mathrm{O}$ autor

Neste caso, os números pares também serão eliminados na primeira rodada, restando apenas $n+1$ soldados. $\mathrm{Na}$ segunda rodada, o primeiro a começar a matar será o soldado de posição 3 , portanto temos uma nova ordem dos soldados para a segunda rodada. 
Tabela 3. Posições após a primeira rodada

\begin{tabular}{l|cccccc} 
Posição: k & 1 & 3 & 5 & 7 & $\ldots$ & $2 n+1$ \\
\hline Nova posição: k' & 0 & 1 & 2 & 3 & $\ldots$ & $n$
\end{tabular}

Fonte: $\mathrm{O}$ autor

ou seja, $k=2 k^{\prime}+1$. A correspondência "posição" e "nova posição" nos dá

$$
J(2 n+1)=2 J(n)+1 \text {. }
$$

Proposição 1: Se o número de soldados no problema de Josephus for uma potência de 2 , ou seja, $n=2^{m}$, então $J(n)=1$.

Dem: Faremos a demonstração por indução finita sobre $m$. Se $m=0$ então $n=1$ e é claro que $J(1)=1$. Suponhamos que o resultado é válido para algum $m \in \mathbb{N}$. Assim,

$$
\begin{gathered}
J\left(2^{m+1}\right)=J\left(2 \cdot 2^{m}\right)=2 J\left(2^{m}\right)-1 \\
=2 \cdot 1-1=1 .
\end{gathered}
$$

Mas não é todo dia que podemos escolher ser refém com um grupo de soldados igual a uma potência de 2 . 0 truque para sobrevir neste caso é justamente partir da potência de 2 , como podemos ver no teorema a seguir.

Teorema 1:

$$
J\left(2^{m}+l\right)=2 l+1,
$$

para $m \geq 0$ e $0 \leq l<2^{m}$.

Dem: A prova deste teorema será feita por indução sobre $m$. Se $m=0$ então

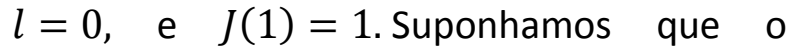
resultado seja válido para algum $m>0$, ou seja $0 \leq l<2^{m}$.

$$
J\left(2^{m}+l\right)=2 l+1, \text { param } \geq 0 \quad \text { e }
$$

Caso 1: $2^{m+1}+l=2 n$

Neste caso temos que $l$ é par, pois $l=2 n-2^{m+1}=2\left(n-2^{m}\right)$, assim $l=2 k$ e obtemos:

$$
\begin{gathered}
J\left(2^{m+1}+l\right)=J\left(2^{m+1}+2 k\right) \\
=J\left(2\left(2^{m}+k\right)\right) \\
=2 J\left(2^{m}+k\right)-1
\end{gathered}
$$

Pelo fato de que $0 \leq l<2^{m+1}$, então $0 \leq 2 k<2^{m+1}$ e portanto $0 \leq k<2^{m}$, podemos assim utilizar a hipótese de indução em (4) e obter:

$$
\begin{gathered}
J\left(2^{m+1}+l\right)=2(2 k+1)-1=2 l+1 . \\
\text { Caso 2: } 2^{m+1}+l=2 n+1 \\
\text { Então, usando }(2), \text { temos que: } \\
\begin{aligned}
J\left(2^{m+1}+l\right) & =J(2 n+1)=2 J(n)+1 \\
& =2+J(2 n),
\end{aligned}
\end{gathered}
$$

mas, $2 n=2^{m+1}+(l-1)$, usando o caso 1 concluímos que

$$
\begin{aligned}
J\left(2^{m+1}+l\right) & =2+J(2 n) \\
& =2+J\left(2^{m+1}+(l-1)\right) \\
& =2+2(l-1)+1 \\
& =2 l+1 .
\end{aligned}
$$

Portanto pelo princípio de indução finita, temos que o teorema é válido para todo $m \in \mathbb{N}$.

\subsection{Números Binários}

O sistema binário, em sua forma geral, adota unicamente dois valores para expressar suas quantias. Estes, são os números " 0 " (zero) e "1" (um) e a partir de tais perspectivas exponha-se o uso das operações elementares (soma, subtração, divisão e multiplicação) com os números binários.

Os números binários são números escritos na base 2 , ou seja, tem somente algarismos 0 (zero) e 1 (um). Em contraste, o sistema numérico decimal (base dez) tem dez valores possíveis $(0,1,2,3,4,5,6,7,8$, ou 9$)$ para cada valor.

3.4.1. Conversão de números decimais em binário

Para converter um número decimal em binário devemos efetuar a divisão por dois, em seguida dividir o quociente dessa divisão, também, por dois, assim sucessivamente. O seu número em binário é $1+$ todos os restos das divisões, do 
quociente menor para o maior, como mostra o exemplo a seguir.

Exemplo:

$$
\begin{aligned}
& 19 \div 2=9 \text { resto } 1 \\
& 9 \div 2=4 \text { resto } 1 \\
& 4 \div 2=2 \text { resto } 0 \\
& 2 \div 2=1 \text { resto } 0 \\
& \text { Portanto, a representação do número }
\end{aligned}
$$
19 na base 2 é 10011.

3.4.2. Conversão de números binários em decimal

Para converter um número binário em decimal, primeiro devemos escrever o número binário $a_{n} a_{n-1} \cdots a_{2} a_{1} a_{0}$, onde os algarismos $a_{i}(i=0, \cdots n)$ é 0 ou 1 , em seguida realizar a multiplicação $a_{n} 2^{n}+a_{n-1} 2^{n-1}+\cdots+a_{2} 2^{2}+a_{1} 2^{1}+a_{0} 2^{0}$. Exemplo:

$$
\begin{aligned}
11001_{2}=1 \cdot 2^{4}+1 \cdot 2^{3}+0 \cdot 2^{2}+0 \cdot 2^{1} \\
+1 \cdot 2^{0}=16+8+1=25 .
\end{aligned}
$$

As operações com números binários seguem o mesmo princípio das operações na base 10 .

\subsubsection{Adição Binária}

Para realizarmos a soma entre dois números binários basta seguirmos a regra a seguir:

Tabela 4. Adição de números binários

Fonte: $\mathrm{O}$ autor

\begin{tabular}{|c|c|}
\hline & Soma \\
\hline $0+0$ & 0 \\
\hline $0+1$ & 1 \\
\hline $1+0$ & 1 \\
\hline $1+1$ & 0 \\
\hline
\end{tabular}

Na soma de 0 e 1 o resultado é 1 . Agora, a soma de 1 com 1 o total é 2 , mas como 2 em binário é 10 , o resultado é o 0 e passa-se 1 para o próximo elemento à esquerda.

Exemplo: $101_{2}+011_{2}$

$$
\begin{gathered}
11 \\
101_{2} \\
+011_{2} \\
\hline 1000_{2}
\end{gathered}
$$

3.4.4 Subtração Binária

Tabela 5. Subtração Binária

\begin{tabular}{|c|c|}
\hline & Subtração \\
\hline $0-0$ & 0 \\
\hline $0-1$ & 1 \\
\hline $1-0$ & 1 \\
\hline $1-1$ & 0 \\
\hline
\end{tabular}

Fonte: $\mathrm{O}$ autor

No caso de 0 (zero) menos 1 (um), temos que "pedir emprestado" do elemento maior. Esse empréstimo vale dois, pois estamos trabalhando com número binário. Logo, no caso de $0-1=1$ pois na verdade a operação feita foi $2-1=1$. E esse processo repete com 0 elemento que faz 0 "empréstimo" e o que valia 1 agora vale 0 .

$$
\text { Exemplo: } \begin{gathered}
101_{2}-011_{2} \\
101_{2} \\
\frac{-011_{2}}{010_{2}}
\end{gathered}
$$

3.4.5 Multiplicação Binária

Tabela 6. Multiplicação Binária

\begin{tabular}{|c|c|}
\hline & Multiplicação \\
\hline $0 \cdot 0$ & 0 \\
\hline $0 \cdot 1$ & 0 \\
\hline $1 \cdot 0$ & 0 \\
\hline $1 \cdot 1$ & 1 \\
\hline
\end{tabular}

Fonte: $\mathrm{O}$ autor

Na multiplicação o número maior deve ser colocado acima do menor e respeitar as operações da Tabela 6 .

$$
\begin{gathered}
\text { Exemplo: } 101_{2} \cdot 011_{2} \\
11 \\
101_{2} \\
\frac{011_{2}}{101} \\
\frac{+1010}{1111_{2}}
\end{gathered}
$$

\subsubsection{Divisão Binária}

Na divisão binária, utiliza-se o mesmo método que a divisão de números decimais: 
Exemplo: $101010_{2} \div 110_{2}$

\begin{tabular}{|c|}
\hline $\begin{array}{c}101010_{2} \\
-110 \downarrow \\
\end{array}$ \\
\hline $\begin{array}{c}1001 \\
-110 \downarrow\end{array}$ \\
\hline $\begin{array}{r}110 \\
-110 \\
\end{array}$ \\
\hline 0 \\
\hline
\end{tabular}

3.5 Problema de Josephus e os números binários

Sempre que ouvimos sobre potências de 2 , a primeira palavra que vem à mente é "binária". A solução para o problema de Josephus é muito mais fácil e mais curta em números binários que em decimais. Apresentaremos uma solução para o problema utilizando números binários.

No problema Josephus havia 40 outros soldados junto com Josephus que faz $n=41.41$ em binário é 101001. Se mudarmos o 1 mais à esquerda para o lugar mais à direita, obtemos 010011 que é 19 (em decimal) que é a resposta. Isso é verdade para uma quantia arbitrária de soldados.

Suponha que temos $n$ soldados, escrevendo em binários temos

$$
\begin{gathered}
n=2^{m}+a_{m-1} 2^{m-1}+\cdots+a_{2} 2^{2}+a_{1} 2 \\
+a_{0}
\end{gathered}
$$

onde $a_{m-1}, \cdots, a_{1}, a_{0} \in\{0,1\}$. Notemos que $n=2^{m}+l$, onde

$$
l=a_{m-1} 2^{m-1}+\cdots+a_{2} 2^{2}+a_{1} 2+a_{0} .
$$

A solução do problema de Josephus é dada por $2 l+1$, ou seja

$$
\begin{gathered}
J(n)=2 l+1=a_{m-1} 2^{m}+\cdots+a_{2} 2^{3} \\
+a_{1} 2^{2}+a_{0} 2+1 .
\end{gathered}
$$

Portanto, em números binários se

então

$$
n=1 a_{m-1} a_{m-2} \cdots a_{1} a_{0},
$$

$$
J(n)=a_{m-1} a_{m-2} \cdots a_{1} a_{0} 1 .
$$

No problema de Josephus temos 41 soldados, vamos converter o número 41 em binário.

$$
\begin{gathered}
41 \div 2=20 \text { resto } 1 \\
20 \div 2=10 \text { resto } 0 \\
10 \div 2=5 \text { resto } 0 \\
5 \div 2=2 \text { resto } 1 \\
2 \div 2=1 \text { resto } 0
\end{gathered}
$$

Portanto se 41 em binário é 101001 , então $J(n)=10011$, representando em decimal temos

$$
\begin{gathered}
1 \cdot 2^{0}+1 \cdot 2^{1}+0 \cdot 2^{2}+0 \cdot 2^{3} \\
+1 \cdot 2^{3}=19
\end{gathered}
$$

Exemplos: Agora vamos aplicar o método com 105 soldados.

Transformando 105 em binário:

$$
\begin{gathered}
105 \div 2=52 \text { resto } 1 \\
52 \div 2=26 \text { resto } 0 \\
26 \div 2=13 \text { resto } 0 \\
13 \div 2=6 \text { resto } 1 \\
6 \div 2=3 \text { resto } 0 \\
3 \div 2=1 \text { resto } 1
\end{gathered}
$$

Portanto 105 em binário é 1101001 , então $J(n)=1010011$, representando em decimal vamos ter

$$
\begin{gathered}
1 \cdot 2^{0}+1 \cdot 2^{1}+0 \cdot 2^{2}+0 \cdot 2^{3}+1 \cdot 2^{4} \\
+0 \cdot 2^{5}+1 \cdot 2^{6}=83
\end{gathered}
$$

\section{DISCUSSÃO}

O Presente trabalho mostrou que podemos obter várias formas de resolver o problema de Josephus por meio da demonstração por indução finita, com isso foi possível criar alguns modelos de resolvê-lo, outra forma é utilizando números binários, com esse método podemos facilitar a resolução do mesmo.

\section{CONSIDERAÇÕES FINAIS}

Este estudo nos proporcionou uma análise do problema de Josephus, que segundo a lenda, para manter-se vivo ele buscou rapidamente um lugar seguro no círculo, assim sendo conforme cada rodada de mortes que acontecia ele se mantinha 
vivo até ser o último, o que resultaria em seu suicídio de acordo com o combinado, no entanto, o objetivo dele não era tirar a própria vida. A partir disso, estudamos um método de demonstração por indução finita no qual nos possibilitou criar uma forma de solução mais rápida. Utilizando os conceitos do conjunto $\mathbb{Z}_{2}$ e ainda o método de demonstração por indução finita, conseguimos criar uma ferramenta que nos permitiu obter a solução imediata deste Problema. Portanto, estas pesquisas nos deram a oportunidade de ampliar a nossa concepção em números binários juntamente com o princípio de indução finita, o que foi de grande relevância para o desenvolvimento deste artigo.

\section{AGRADECIMENTOS}

Agradecimentos à revisores, colaboradores e agências de fomento

\section{REFERÊNCIAS}

FOMIN, D.; GENKIN, S.; ITENBERG, I. Círculos matemáticos - a experiência Russa: Rio de Janeiro: Impa, 2010.

HEFEZ, A. Indução Matemática. Rio de Janeiro: OBMEP, 2009.

MALAGUTTI, P. L. SAMPAIO, J, C. Mágicas, matemática e outros mistérios. São Carlos: UFSCar, 2012.

OLIVEIRA, K. I. M.; FERNANDEZ, A. J. C. Iniciação à matemática: um curso com problemas e soluções. Rio de Janeiro: SBM, 2010.

SANTOS, J. P. O.; MELLO, M. P.; MURARUI, I. T. C. Introdução à análise combinatória: Rio de Janeiro, Ciência Moderna, 2007.

WINTER, O. C. Relações de recorrências: para além de P.A e P.G. Dissertação (Mestrado em
Matemática) - Universidade Federal do ABC, Santo André, 2013. 